\title{
KINETIC MONTE CARLO STUDY OF STRUCTURAL PROTON DIFFUSION ALONG A ONE-DIMENSIONAL PATH
}

\author{
Tomasz Masłowski, Andrzej Drzewiński, Małgorzata Zubaszewska* \\ University of Zielona Góra, Institute of Physics, Zielona Góra, Poland \\ "mzubaszewska@o2.pl
}

\begin{abstract}
We study the structural diffusion coefficient of protons in a chain-like system by the means of KMC simulations. To perform specific calculations, we adopted parameter values in our model that correspond to the benzimidazole crystal. As we have shown, the structural diffusion follows the Arrhenius-like thermal dependence with an effective activation energy of $2.01 \mathrm{eV}$.
\end{abstract}

Keywords: computational statistical mechanics, diffusion processes

\section{Introduction}

Diffusion can be described as a stochastic process where a single particle can move in each direction with the same probability [1]. In one dimensional, the mean square displacement of such particle is given by the formula $\left\langle r^{2}\right\rangle=2 D t$. In this study, the diffusion of protons is under consideration. The symbol $\left\langle r^{2}\right\rangle$ denotes the square of the distance separating the proton position at $t=0$ from its position at time $t$, averaged over all the protons in the system, whereas $D$ is the diffusion coefficient. The variable $\left\langle r^{2}\right\rangle$ can be interpreted as the statistical variance of the particle positions where $D$ measures the rate of variance change.

The diffusion coefficient at different temperatures is expected to have the Arrhenius form

$$
D=D_{0} \exp \left(\frac{-E_{a}}{k_{B} T}\right)
$$

where $T$ is the absolute temperature, $k_{B}$ is the Boltzmann constant, $D_{0}$ is the maximum diffusion coefficient and $E_{a}$ is the activation energy. This last quantity can be deduced from a linear fit of the lin-log plot.

The structural proton diffusion is a specific phenomenon in many organic or inorganic compounds [2]. Namely, based on the Grotthuss mechanism, it requires two processes: proton hopping from one anionic group to the next, and also a reorientation of proton-carrying anionic groups (see Fig. 1). It is characterized by 
the separation of time scales between rotations and hops that results from the necessity to break and create hydrogen bonds ( $H$-bonds). This type of dynamics is called a rare-event dynamics, which means that the time between consecutive events can be orders of magnitude longer than the actual process time itself. The simulation technique that handles such behavior is the kinetic Monte Carlo (KMC) that enables us to combine an accurate description of the elementary processes with an account for their statistical interplay in order to properly evaluate the proton dynamics $[3,4]$.

As it has been shown [2], the structural diffusion (Grotthuss mechanism) is more effective than proton transport via molecular diffusion when the molecule acts as a vehicle to transport the proton (so-called vehicle mechanism). For example, it is commonly accepted that the abnormally high proton mobility in water is a consequence of the Grotthuss mechanism.

The question arises whether for the structural diffusion the Arrhenius formula is still valid.

\section{Model}

As the model system, we propose a chain of parallel rigid rods whose ends can be occupied by protons, one proton per end. Rods with or without protons can independently rotate by $180^{\circ}$. Protons can also migrate by hopping from one rod to the nearest one provided the end of the adjacent rod is empty (Fig. 1). Each KMC trajectory consists of a sequence of discrete transitions from one system state to another. The probabilities prescribed by the Master Equation allow us to determine the lifetime of a particular state and select the next state. The key point is to know a priori all transition rates from every configuration to any other allowed one.

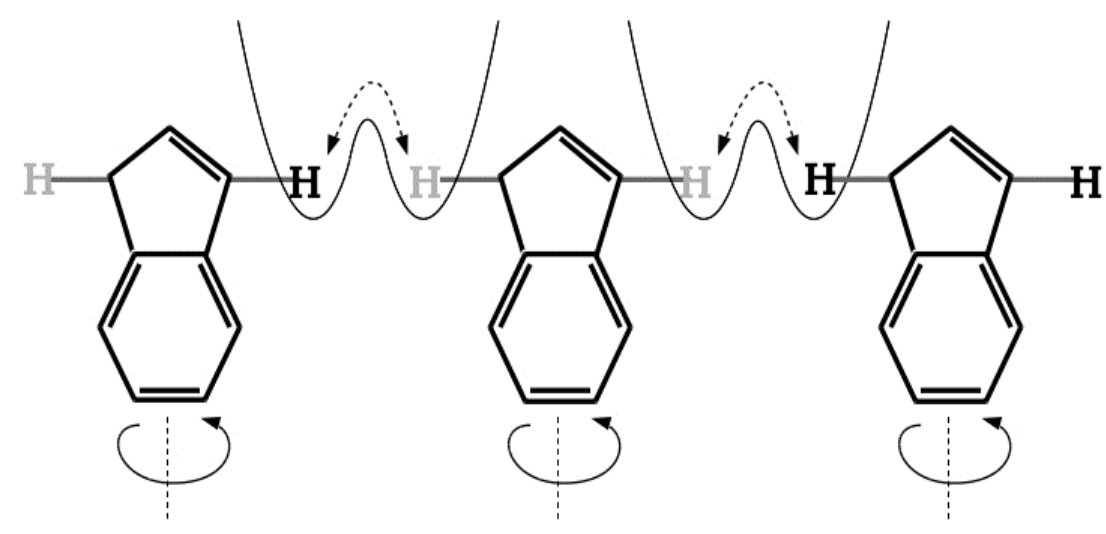

Fig. 1. The pathway of a charge transfer where solid Hs represent protons. The dashed arrow represents proton hops within a H-bond double well potential. The solid arrows correspond to the benzimidazole rotations. The periodic boundary conditions permit hops between the leftmost and rightmost rods 
In order to perform specific calculations, we have adopted in our model parameter values that correspond to the benzimidazole $\left(\mathrm{C}_{7} \mathrm{H}_{6} \mathrm{~N}_{2}\right)$. According to impedance spectroscopy and $1 H$ NMR experimental results [5] the proton conduction process of the benzimidazole follows the Grotthuss mechanism. It involves both molecular $180^{\circ}$ flips prior to the proton exchange and migration along the hydrogen bonded chain via the $N-H \cdots N$ bridges. As the $H$-bond is almost linear (the angle $\left.\left(N H N=172^{\circ}\right)[6]\right)$. In the previous paper we have studied the proton conductivity in polymer electrolyte membranes based on benzimidazole molecules [7].

\section{Method}

Focusing on this Markovian state-to-state dynamics by hops is the central idea behind the KMC simulations. Therefore, in the KMC method, one uses transition rates that depend on the energy barrier between the states $[7,8]$.

Herein, the rotations are treated as a thermally activated process satisfying the Arrhenius law with the activation energy $V_{a c t}$. Then the frequency of rotation is given by

$$
v_{R}=v_{0} \exp \left(-V_{a c t} / k_{B} T\right)
$$

During the proton transmission, it is possible that a proton is supplied to the $H$-bond, where another proton is present. Such a configuration is energetically unfavorable as well as the configuration with the empty $H$-bond because of interacting electron clouds. These effects can be included by introducing an additional Boltzmann factor with the energy penalty $V_{\text {Coul }}$. So, finally the transition rate for rotations becomes

$$
\gamma_{R}=v_{R} \exp \left(-V_{\text {Coul }} / k_{B} T\right)
$$

The migration of a proton from one rod to another represents the hopping between the minima of the $H$-bond potential. Hopping is defined as the thermally assisted tunneling which is an extension of the purely classical Arrhenius behavior. The tunneling rate is calculated using Bell's formula [9]

$$
\begin{gathered}
\tau_{T}=\frac{1}{k_{B} T} \int_{0}^{\infty} \mathrm{G}(\mathrm{E}) \exp \left(\frac{-E}{k_{B} T}\right) d E, \\
G(E)= \begin{cases}1 /\left[1+G_{W K B}^{-1}\right], & \text { for } E \leq V_{\max } \\
1, & \text { for } E>V_{\max }\end{cases}
\end{gathered}
$$

where

$$
G_{W K B}(E)=\exp \left(\frac{-2}{\hbar} \int_{z_{1}(E)}^{z_{2}(E)} \sqrt{2 m[V(z)-E]} d z\right)
$$


is the WKB quantum permeability of the proton with energy $E$. It travels between classical turning points $z_{1}(E)$ and $z_{2}(E)$ of the potential $\mathrm{V}(z)$ approximated by the fuzzy Morse potentials originating in rod ends as they represent anionic groups between which the $H$-bonds are created.

$$
\begin{gathered}
\mathrm{V}(z)=\frac{1}{2 a} \int_{-a}^{a}\left[V_{\text {Morse }}\left(\frac{d}{2}-z+y\right)+V_{\text {Morse }}\left(z-y-\frac{d}{2}\right)\right] d y, \\
V_{\text {Morse }}(z)=g\left[\exp \left(\frac{-2 z}{b}\right)-2 \exp \left(\frac{-z}{b}\right)\right] .
\end{gathered}
$$

The parameter $d$ refers to the bond length while the parameter $a$ controls the dispersion in the position of the nitrogen ions forming the $H$-bond and represents the lattice vibrations. The parameters $g$ and $b$ are fitted to get the respective distance between the minima of the double-well potential $V(z)$ together with the height of the barrier. Finally, the total hopping rate becomes

$$
\gamma_{T}=v_{T} \tau_{T}
$$

where $v_{T}$ is the hopping frequency prefactor.

Since we are going to analyze thermal properties, we need to insert them properly into the model, not only through the scale of the thermal energy $k_{B} T$. That is why, using the Taylor series expansion at a reference temperature $T_{0}$, we consider the thermal dependencies of the $a$ and $d$ parameters where the higher order terms can be neglected.

$$
\begin{aligned}
& a(T)=a_{0}+a_{1}\left(T-T_{0}\right), \\
& d(T)=d_{0}+d_{1}\left(T-T_{0}\right) .
\end{aligned}
$$

\section{Results}

Values of parameters for the benzimidazole simulations are as follows [7]: frequency of rotation prefactor $v_{0}=10^{12} \mathrm{~Hz}$, activation energy for rotations $V_{a c t}=0.269 \mathrm{eV}$, rods length $b=3.84 \AA$, bond length $d_{0}=2.886 \AA$, thermal expansion coefficient $d_{1}=1.1 \times 10^{-5} \AA / \mathrm{K}, V(z)$ barrier height $h\left(T_{0}\right)=0.38 \mathrm{eV}$, distance between minima of $V(z), \Delta z\left(T_{0}\right)=0.77 \mathrm{eV}$, reference temperature $T_{0}=393 \mathrm{~K}$, Coulomb energy penalty $V_{\text {Coul }}=0.04 \mathrm{eV}$, frequency of hopping prefactor $v_{T}=10^{9} \mathrm{~Hz}$, lattice vibration amplitude $a_{0}=0.2 \AA$, and thermal susceptibility of $a_{0}=0.002 \AA / \mathrm{K}$. It is worth stressing that most of the parameters have been estimated on the basis of experimental data or the quantum-mechanical calculations. 


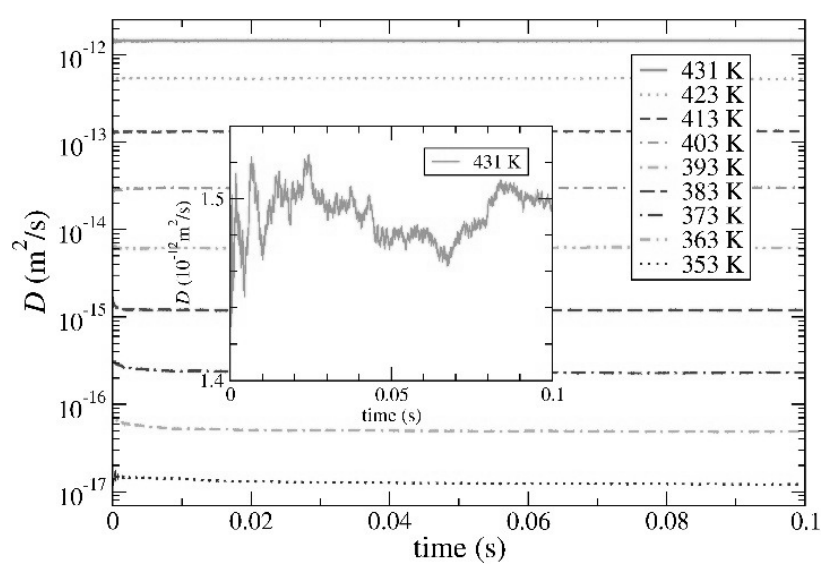

Fig. 2. The structural diffusion coefficient for various temperatures. The inset shows an enlargement of an individual simulation

In the present calculations the system of $N=200$ rods, which implies the same number of protons, has been considered. At each temperature the entire KMC simulation is divided into $n=100$ simulations where initial configurations are chosen randomly and long proton trajectories are generated. To figure out the diffusion coefficient the following formula is applied

$$
D=\frac{1}{2 N t}\left\langle\sum_{i=1}^{N} r_{i}^{2}(t)\right\rangle,
$$

where the index $i$ enumerates individual protons. The angle brackets represent averaging over independent trajectories. The temperature-dependent number of KMC steps needed to reach time 0.1 seconds varies from $2 \cdot 10^{9}$ to $7.5 \cdot 10^{9}$ (for one simulation).

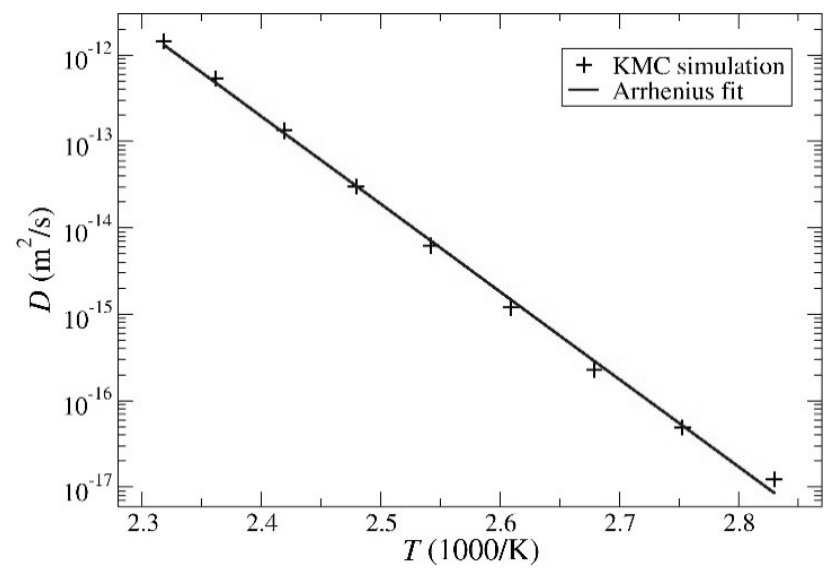

Fig. 3. Temperature dependence of the diffusion coefficient determined by the KMC simulation (the crosses). The solid line presents the best linear fit to the numerical data 
The structural diffusion coefficients were computed from the linear fit between $353 \mathrm{~K}$ to $431 \mathrm{~K}$. According to our analysis, its temperature dependence shows an Arrhenius dependence on temperature with an activation energy of $2.01 \mathrm{eV}$.

\section{Conclusions}

It has been examined that our numerical model can describe the proton diffusion based on the Grotthuss mechanism. To perform specific calculations, we adopted the parameter values corresponding to the benzimidazole crystal.

The straight line fit in the temperature region studied confirms that the temperature-dependent structural diffusion coefficient demonstrates the expected Arrhenius dependence on temperature with an effective activation energy of $2.01 \mathrm{eV}$.

\section{Acknowledgment}

M.Z. is a scholar within Sub-measure 8.2.2 Regional Innovation Strategies, Measure 8.2 Transfer of knowledge, Priority VIII Regional human resources for the economy Human Capital Operational Programme co-financed by the European Social Fund and state budget.

\section{References}

[1] Shewmon P.G., Diffusion in Solids, McGraw-Hill, New York 1963.

[2] Kreuer, K.D., Proton conductivity - materials and applications, Chem. Mater. 1996, 8, 610-641.

[3] Bortz A.B., Kalos M.H., Lebowitz J.L., A new algorithm for Monte Carlo simulation of Ising spin systems, J. Comput. Phys., 1975, 17, 10-18.

[4] Hermet J., Bottin F., Dezanneau G., Geneste G., Kinetic Monte Carlo study of protonic diffusion and conduction in Gddoped $\mathrm{BaCeO}_{3}$, Solid State Ionics 2013, 252, 48-55.

[5] Zdanowska-Fraczek M., Hołderna-Natkaniec K., Ławniczak P., Pawlaczyk C., Molecular dynamics and electric conductivity process efficiency in an anhydrous system. ${ }^{1} \mathrm{H}$ NMR study of benzimidazolium azelate, Solid State Ionics 2013, 237, 40-45.

[6] Krawczyk S., Gdaniec M., Polymorph $\beta$ of $1 H$-benzimidazole, Acta Crys. E 2005, 61, 4116-4118 .

[7] Masłowski T., Drzewiński A., Ulner J., Wojtkiewicz J., Zdanowska-Frączek M., Nordlund K., Kuronen A., Kinetic Monte Carlo simulations of proton conductivity, Phys. Rev. E 2014, 90, 012135-1-012135-8.

[8] Masłowski T., Drzewiński A., Lawniczak P., Ulner J., Numerical modeling of the heterocycle intercalated proton-conducting polymers at various mole ratios, Solid State Ionics 2015, 272, 166-169.

[9] Bell R.P., The application of quantum mechanics to chemical kinetics, Proc. R. Soc. London ser. A $1933,139,466-474$. 